\title{
Synchrotron investigation on the sheared structure evolution of syndiotactic polypropylene crystallization process
}

\section{$\operatorname{AUTHOR(S):~}$}

Wen, Huiying; Jiang, Shichun; Men, Yongfeng; Zhang, Xuequan; An, Lijia; Wu, Zhonghua; Okuda, Hiroshi

\section{CITATION:}

Wen, Huiying ...[et al]. Synchrotron investigation on the sheared structure evolution of syndiotactic polypropylene crystallization process. JOURNAL OF CHEMICAL PHYSICS 2009, 130(16): 164909.

\section{ISSUE DATE:}

2009-04

URL:

http://hdl.handle.net/2433/109907

\section{RIGHT:}

Copyright 2009 American Institute of Physics. This article may be downloaded for personal use only. Any other use requires prior permission of the author and the American Institute of Physics. The following article appeared in JOURNAL OF CHEMICAL PHYSICS 130, 164909 (2009) and may be found at http://link.aip.org/link/JCPSA6/v130/i16/p164909/s1 


\title{
Synchrotron investigation on the sheared structure evolution of syndiotactic polypropylene crystallization process
}

\author{
Huiying Wen, ${ }^{1,2}$ Shichun Jiang, ${ }^{1,3, a)}$ Yongfeng Men, ${ }^{1}$ Xuequan Zhang, ${ }^{1}$ Lijia An, ${ }^{1, a)}$ \\ Zhonghua Wu, ${ }^{4}$ and Hiroshi Okuda ${ }^{5}$ \\ ${ }^{1}$ State Key Laboratory of Polymer Physics and Chemistry, Changchun Institute of Applied Chemistry, \\ Chinese Academy of Sciences, Changchun 130022, People's Republic of China \\ ${ }^{2}$ College of Engineering and Technology, Northeast Forestry University, Harbin 150040, \\ People's Republic of China \\ ${ }^{3}$ School of Materials Science and Engineering, Tianjin University, Tianjin 300072, \\ People's Republic of China \\ ${ }^{4}$ Beijing Synchrotron Radiation Laboratory, Institute of High Energy Physics, Chinese Academy \\ of Sciences, Beijing 100039, People's Republic of China \\ ${ }^{5}$ International Innovation Center, Kyoto University, Sakyo-ku, Kyoto 606-8501, Japan
}

(Received 7 September 2008; accepted 3 April 2009; published online 29 April 2009)

\begin{abstract}
The final structure of molten syndiotactic polypropylene (sPP) sheared under different conditions was investigated by synchrotron small-angle x-ray scattering (SAXS) and wide-angle x-ray diffraction (WAXD) techniques to elucidate the shear effects on sPP crystalline structure. The results obtained from the WAXD show that there is no variation on crystalline form but a little difference on the orientation of the 200 reflection. The SAXS data indicate that the lamellar thickness and long period have not been affected by shear but the lamellar orientation is dependent on shear. The experimental data of sPP crystallization from sheared melt may indicate a mesophase structure that is crucial to the shear effects on the final polymer multiscale crystalline structures. (C) 2009 American Institute of Physics. [DOI: 10.1063/1.3125383]
\end{abstract}

\section{INTRODUCTION}

The Hoffman-Lauritzen (LH) theory of secondary, surface nucleation, and growth were relied for a long time. ${ }^{1}$ The theory assumes that the growth of lamellae is formed by the direct attachment of chain sequences from the melt onto smooth lateral sides and the lamellar thickness is determined by the supercooling below the equilibrium melting point given by the Gibbs-Thomson equation. The LH model was confronted by criticism and some of the points were taken up and led to modifications, ${ }^{2-4}$ but the foundation remained unchanged which deemed that before the nucleation and crystallization the system was uniform. Whereas, more and more experiment results show that before the crystallite appears polymer chains go through conformation adjustment and orientation transformation which favor the formation of crystallites, i.e., a preordering structure exist before or at the early stage of polymer crystallization. ${ }^{5-18}$ This is a deviation from the simple process of nucleation and growth at the early stages of melt crystallization which can be gone back to Ostwald's "rule of stages." 19 This rule states that crystals always nucleate into the mesomorphic or crystalline structure which is the metastable one for nanometer-size crystals and differ from the crystal modification which is macroscopically stable.

Even if all the examples include crystallization from the transient intermediate phase, the mechanism about amorphous phase of flexible chain molecules transforms into semicrystalline state is still a puzzle. Studies of polymer

${ }^{a)}$ Electronic addresses: scjiang@tju.edu.cn and ljan@ciac.jl.cn. crystallization require an understanding based on information of the molecular processes controlling the transformation. On the base of observations and experiments, Strobl and co-workers ${ }^{20-26}$ proposed a different model to elucidate polymer crystallization process despite the fact that it was not been commonly accepted. The major components of this model are the concept of the formation of a mesomorphic melt as precursor to crystallization and the control of the melting temperature range of lamellar crystals of homogeneous polyolefin copolymers by ab inner degree of order or perfection rather than on the crystal thickness. It is proposed that the initial step is always the creation of a mesomorphic layer which spontaneously thickens up to a critical value where the stable crystal phase is favored and it solidifies through a cooperative structural transition. The transition produces a granular crystalline layer, which transforms in the last step into homogeneous lamellar crystallites. The assumption is solidified by observations showing granular substructure for many semicrystalline polymers. ${ }^{27-34}$ Strobl and co-workers ${ }^{32-34}$ found that syndiotactic polypropylene (sPP) crystallization is a two-step process by atomic force microscopy (AFM) observation, with the block formation, i.e., a granular substructure near the growth front as a first and their fusion into a lamella as a second step. Lately based on Strobl's suggestion, some researchers probed into mesophase forming polymers. ${ }^{35,36} \mathrm{We}$ have investigated sPP crystallization process from sheared melt by time-resolved synchrotron small-angle x-ray scattering (SAXS) techniques, and the obtained experimental dada convinced us that there is a stable mesophase before shear is composed. ${ }^{37}$ At present, the model promoted by Strobl and co-workers appears to be at an un- 
certain stage at which strong proof or disproof are not available. However, this alternative model has injected a new vitality into the study of crystallization of flexible chain polymers. We believe that the crystallization framework proposed by Strobl is generally feasible for many situations. In this report, the evidence confirms that the polymer crystallization begins with a transient mesophases and continuously explores the stability of the transient mesomorphic phase under shear.

sPP was all along put less interest than its isotactic counterpart (isotactic polypropylene) for its low crystallinity and lower regio- and stereoregularities. Since Ewen et al. ${ }^{38,39} \mathrm{ob}-$ tained highly steroregulatities syndiotactic form by metallocene catalysts, this polymer has gained further market and led to more interest in scientific research and industrial applications. ${ }^{4-48}$ For semicrystalline polymers, the structure and morphology after processing can directly affect physical and mechanical properties of the final products. Some studies have focused on the fact that semicrystalline polymers crystallized under applied field such as shear field has an increasing crystallization dynamics, different crystalline morphologies and mechanical properties compared to polymer under quiescent conditions. ${ }^{4-55}$ Although some researches have been carried out on the crystalline structure and morphology of sPP, only few reports considered the influence of shear on the crystallization structure of sPP. It is now clear that $\mathrm{sPP}$ exhibits a granular substructure which has been directly observed by AFM. How does the ordered phase to final crystallize undergo from the amorphous melt and what the function of shear on this process. In this work a track from the final crystalline structure under different shear conditions has been performed. wide-angle x-ray diffraction (WAXD) and synchrotron SAXS techniques were used to investigate the structure of SPP under various crystallization temperatures and shear conditions. This work aims at tracing the detailed information of the effect of shear on the structure for sPP to understand the early stage of crystallization process.

\section{EXPERIMENTAL SECTION}

\section{A. Sample characteristics and preparation}

The sample of sPP is a commercial product obtained from FINA Oil, Brussels. It has $83 \%$ syndiotactic pentades and a molar mass $M_{w}=6 \times 10^{4} \mathrm{~g} \mathrm{~mol}^{-1}\left(M_{w} / M_{n}=4\right)$. A Linkam CSS 450 shear cell was used to control the temperature and shear rate and the sample was fixed by two quartz slices. The sample was first heated to $200{ }^{\circ} \mathrm{C}$ with a rate of $30 \mathrm{~K} / \mathrm{min}$ and maintained at $200{ }^{\circ} \mathrm{C}$ for $5 \mathrm{~min}$ to eliminate any residual effects, then cooled to crystallization temperature with a rate of $-30 \mathrm{~K} / \mathrm{min}$. During this process, a $5 \mathrm{~s}$ step shear was applied to the sample as soon as the isothermal crystallization temperature was reached. After crystallization, the thickness of the sample was about $1000 \mu \mathrm{m}$.

\section{B. Measurements}

WAXD spectra were obtained with a Rigaku D/max 2500 V PC x-ray diffractometer (Japan) with a $\mathrm{Cu} K \alpha 1$ source working at $40 \mathrm{kV}$ and $200 \mathrm{~mA}$. The scattering angle

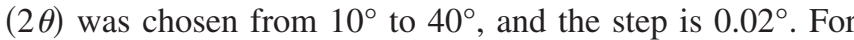

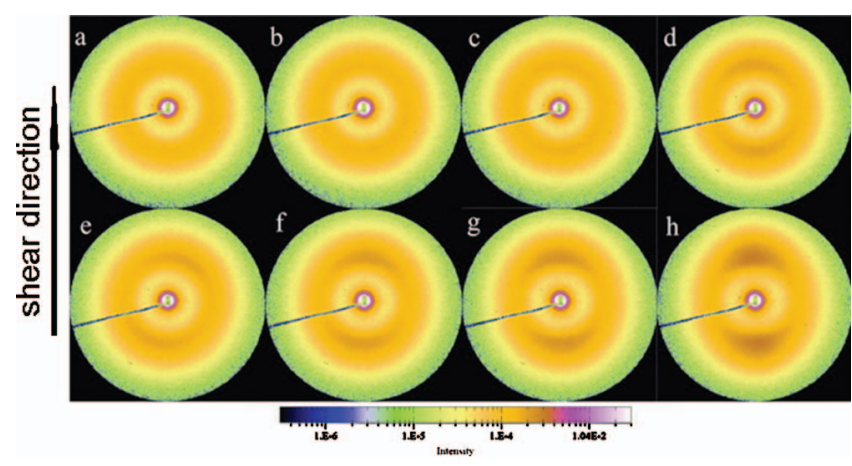

FIG. 1. (Color) 2D SAXS patterns of sPP crystallized at $100{ }^{\circ} \mathrm{C}$ with different shear rates. (a) $0 \mathrm{~s}^{-1}$, (b) $0.2 \mathrm{~s}^{-1}$, (c) $0.5 \mathrm{~s}^{-1}$, (d) $1 \mathrm{~s}^{-1}$, (e) $2 \mathrm{~s}^{-1}$, (f) $5 \mathrm{~s}^{-1}$, (g) $10 \mathrm{~s}^{-1}$, and (h) $20 \mathrm{~s}^{-1}$.

two-dimensional (2D) WAXD, an imaging plate with a dimension of $127 \times 127 \mathrm{~mm}^{2}$ provided by Fuji Photo Films Co. Ltd was used. The exposure time was $60 \mathrm{~min}$.

The SAXS measurements on the shear-induced structure of sPP were conducted at Synchrotron beam line BW4 at HASYLAB, Hamburg, Germany and synchrotron beam line BL15A of the photon factory of the High Energy Accelerator Research Organization (KEK), Tsukuba, Japan. The x-ray wavelength at Hamburg is $1.38 \AA$ and the $\mathrm{x}$-ray wavelength at BL15A is $1.54 \AA$. All of the data were corrected for background scattering before analysis and treated with the software FIT 2D. ${ }^{56}$

\section{RESULTS AND DISCUSSION}

It was thought that the SAXS peak resulted from density fluctuations on the order of 5-30 nm which basically covers the size of lamellar structure of crystalline polymers, while WAXS crystalline peaks were brought about by threedimensional crystal ordering on the order of $0.2-1 \mathrm{~nm}$ which correspondingly covers the range of crystallite of crystalline polymers. Their association can depict the crystalline forms and crystallization microstructure of the semicrystalline polymer very well.

Figure 1 shows the synchrotron SAXS patterns of sPP crystallized at $100{ }^{\circ} \mathrm{C}$ after $5 \mathrm{~s}$ shearing with different shear rate. The SAXS image obtained without shear (with $180^{\circ}$

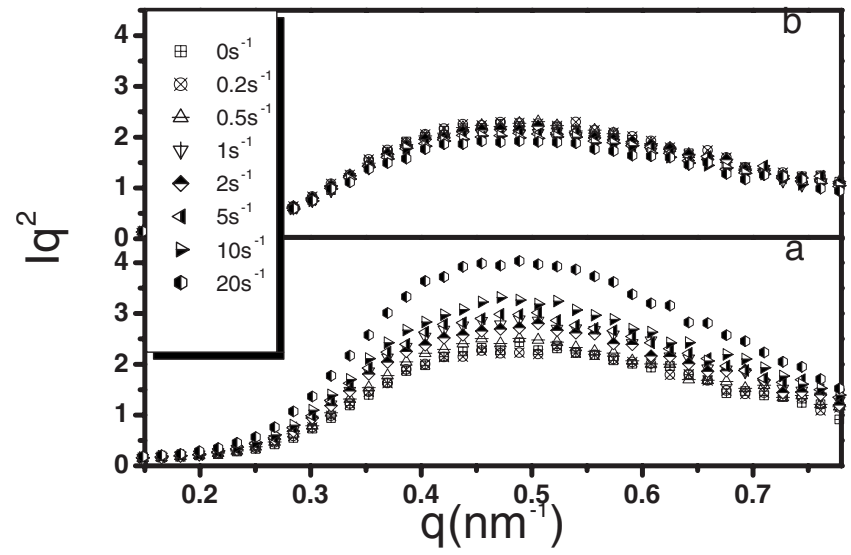

FIG. 2. $I q^{2}$ vs scattering vector $q$ of sPP after isothermal crystallization at $100{ }^{\circ} \mathrm{C}$ with different shear rates along the meridian. 


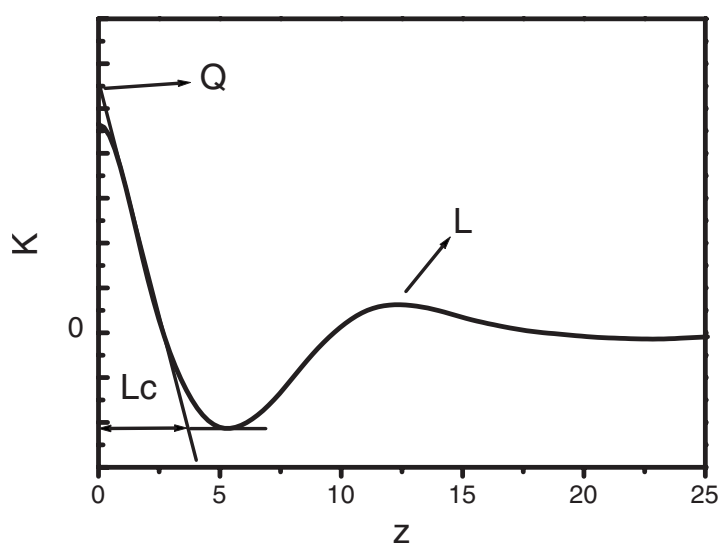

FIG. 3. Schematic drawing showing the basic properties of the electron density correlation function $K(z)$ associated with a stack of crystalline and amorphous layers. $K(z)$ derived from the scattering curve of sPP crystallized under shear rate $20 \mathrm{~s}^{-1}$ in Fig. 1, giving $L=12.4 \mathrm{~nm}$ and $L c=3.4 \mathrm{~nm}$.

rotational symmetry) indicates that crystalline lamellae are randomly distributed. As the shear rate increases, the pattern varies as shown in Fig. 1(c) where weak orientation is observed. The degree of orientation for scattering pattern increases with the shear rate increasing. Lorentz corrected intensity $I q^{2}$ as a function of the scatter vector $q$ under different shear intensities along the meridian (shear direction) and the equator (perpendicular to shear direction) is shown in Fig. 2. As is well known, the intensity of SAXS is dependent on the electron density difference between the phases of the system. With the increasing shear rate, the scattering intensity along the meridian became stronger whereas the intensity along the equator decreased slightly. We suppose that there exist more amount of or perfect crystalline structures which are responsible for higher value of the electron density difference on the meridian with shear composed. Under shear, the peak position $q_{\max }$ does not change either along the meridian or along the equator, as indicated in Fig. 2. According Bragg's law, $L=2 \pi / q_{\max }$, where the $q_{\max }$ represents the peak position in the Lorentz corrected scattering intensity plot, $L$ is the long period between the adjacent layers (lamellae). The results in Fig. 2 showed that the long period is not affected by shear, even the scattering intensity

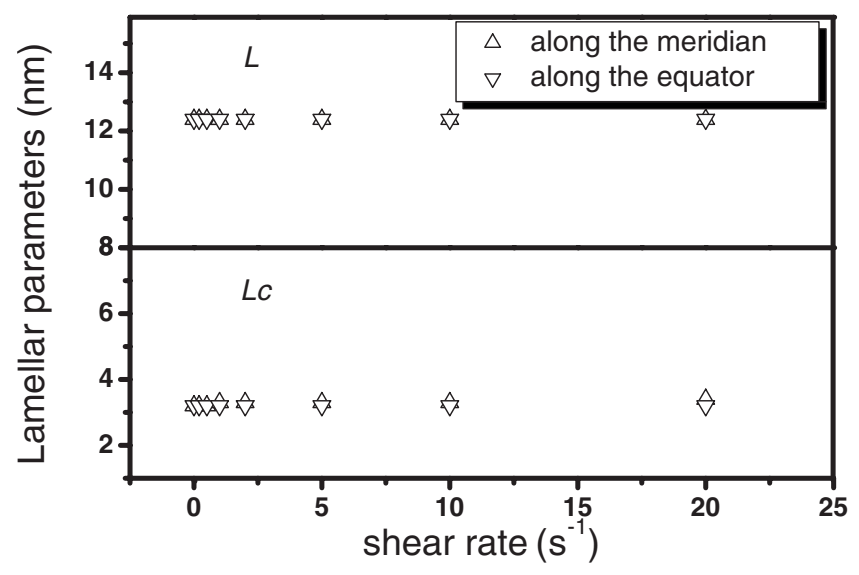

FIG. 4. Lamellar parameters: Long period $(L)$, crystal lamellar thickness $(L c)$ obtained from SAXS data of SPP after isothermal crystallization at $100{ }^{\circ} \mathrm{C}$ as a function of shear rate.

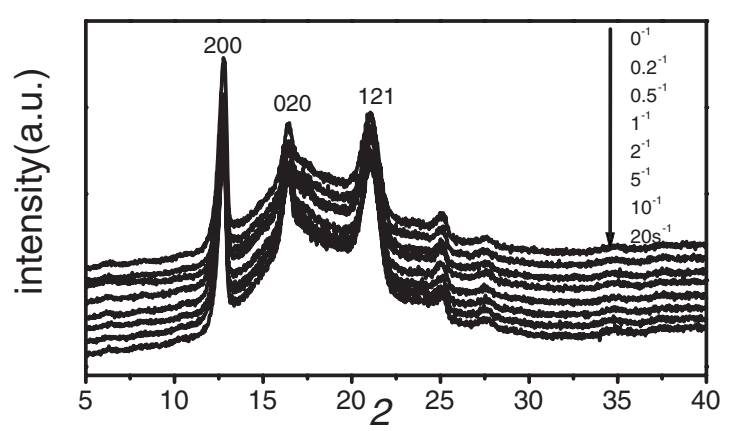

FIG. 5. 1D WAXD intensity profiles of sPP with various shear rate after isothermal crystallization at $100{ }^{\circ} \mathrm{C}$.

is related to the shear rate. One can make a conclusion that long period in sPP is not determined by shear rate.

To obtain detail information about structure parameters such as long period and average lamellar thickness, the onedimensional (1D) electron intensity correlation function $K(z)$, which can be derived from Fourier transform of the scattering curve, was adopted to learn the influence of shear on the microstructure, ${ }^{57}$

$$
K(Z)=\frac{1}{2 \pi} \int_{0}^{\infty} I(s) s^{2} \cos (s Z) d s,
$$

where $s=2 \sin \theta / \lambda=q / 2 \pi$, the modulus of the momentum transfer vector $q$. Many studies have reported that the value of the long period calculated by Bragg's law $L_{b}$ is always a little larger than $L$ calculated from the correlation function. ${ }^{58-61}$ This phenomenon is common for many semicrystalline polymers and may be the presence of a broad distribution in long period. There is a broader distribution of long periods in scattering curve due to the existence of tran-

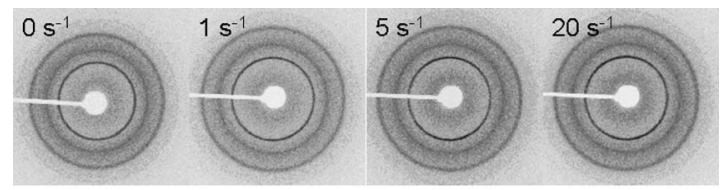

(a)

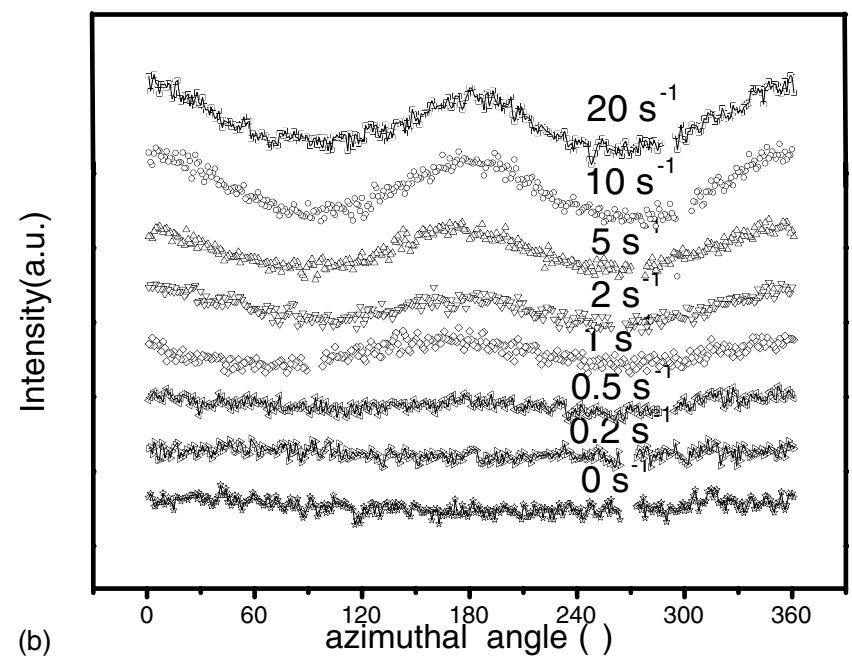

FIG. 6. (a) Selected 2D WAXD patterns and (b) azimuthal intensity curves of (200) reflections of sPP after isothermal crystallization at $100{ }^{\circ} \mathrm{C}$ with different shear rates. 


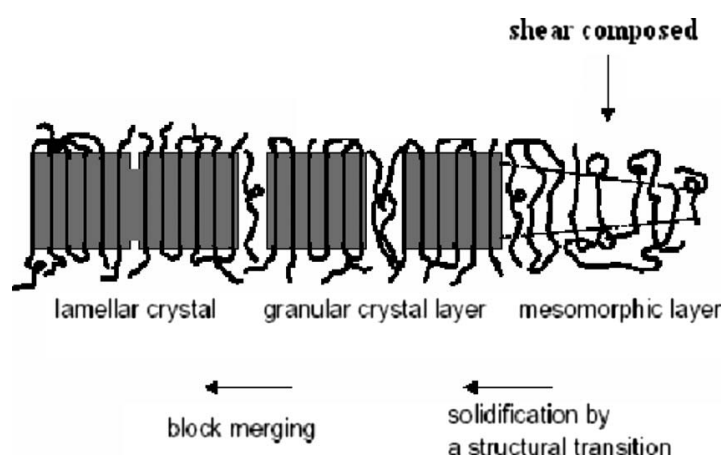

FIG. 7. Schematic representation of the route as suggested in Ref. 25 proposed for the formation of sPP crystallite from a mesomorphic layer to a final lamellar layer with shear composed.

sition zone that makes the peak position $q_{\max }$ in the Lorentz corrected scattering plot moving to the lower value. It must be mentioned that for a model of lamellar stacks the correlation function containing the basic morphological information is based on the unoriented samples of a partially crystalline polymer where the lamellae structure is isotropically distributed. Some theory was placed forward that no multiplication $s^{2}$ to $I(s)$ is performed for the completely oriented system. $^{62-64}$ In our experiment the structure parameters of partially oriented lamellae with anisotropic intensity distribution would be calculated by correlation function of Eq. (1). Figure 3 shows schematic drawing of the electron density correlation function $K(z)$ derived from the scattering curve of sPP crystallized at shear rate $20 \mathrm{~s}^{-1}$. We discuss the lamellar structure parameters both along the equator and the meridian separately which would represent the average values of the lamellae orientated in the shear direction and in the direction perpendicular to shear direction, respectively. Figure 4 shows the detailed structural parameters for sPP after isothermal crystallization at $100{ }^{\circ} \mathrm{C}$ with different shear rates in both directions calculated from Eq. (1). The long period $L$ does not have any change and the lamellar thickness $L c$ almost remains invariant either along the meridian or along the equator with the increase of the shear rate.

Knowing the effects of shear on the lamellar structure, one may wonder how the shear will influence the crystalline

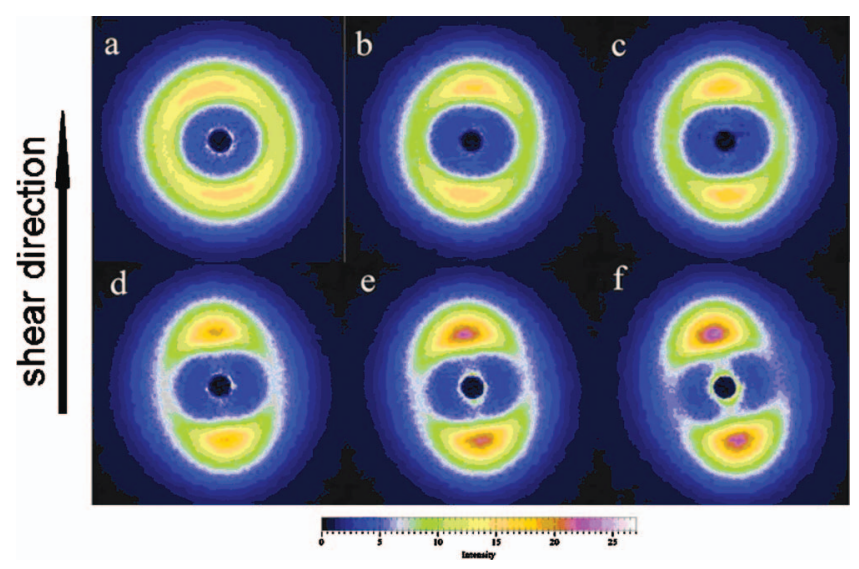

FIG. 8. (Color) 2D SAXS patterns of sPP after isothermal crystallization at $100{ }^{\circ} \mathrm{C}$ with different shear duration. (a) $5 \mathrm{~s}$, (b) $10 \mathrm{~s}$, (c) $20 \mathrm{~s}$, (d) $40 \mathrm{~s}$, (e) $80 \mathrm{~s}$, and (f) $120 \mathrm{~s}$.

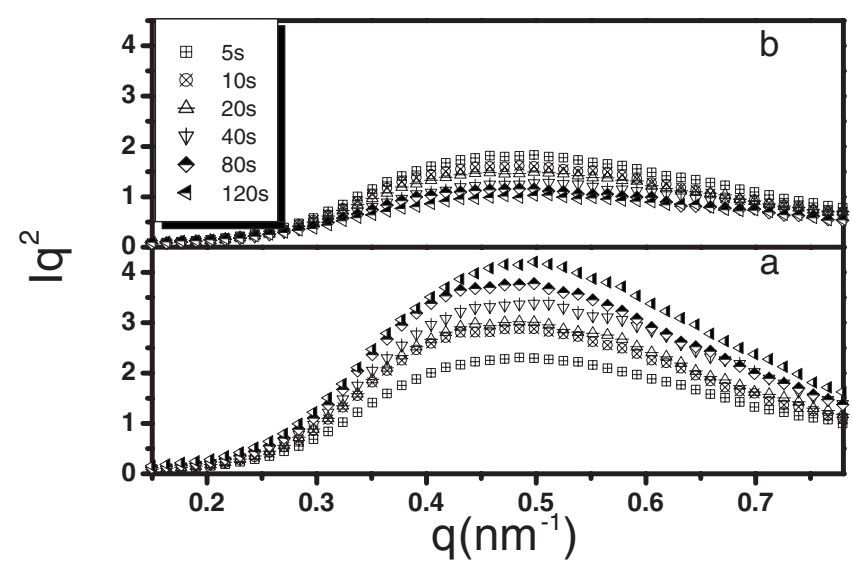

FIG. 9. $I q^{2}$ vs scattering vector $q$ of sPP after isothermal crystallization at $100{ }^{\circ} \mathrm{C}$ with different shear durations under fixed shear intensity $5 \mathrm{~s}^{-1}$. (a) Along the meridian; (b) along the equator.

structure of sPP? Figure 5 shows the 1D WAXD patterns of sPP isothermal crystallized at $100{ }^{\circ} \mathrm{C}$ with different shear rates for $5 \mathrm{~s}$ shearing at $100{ }^{\circ} \mathrm{C}$ before crystallization. The peak at $2 \theta=12.6^{\circ}$ is corresponding to the 200 reflections; the other peaks at $2 \theta=16.4^{\circ}$ and $2 \theta=21.0^{\circ}$ are close to the positions expected for the 020 and the 121 reflections. As is known, sPP has three types of crystalline forms: forms I, ${ }^{65-69}$ II, ${ }^{70,71}$ and III. ${ }^{72,73}$ In the three crystalline forms, form I is considered to be the most stable one which has a $T_{2} G_{2}$ chain conformation setting up a helix with two syndiotactic units per turn and always obtained by melt crystallization. Two orthorhombic forms have been reported for form I. The better symmetry called limit-ordered structure of sPP has a unit cell which includes two left- and two right-handed helices in a regular alteration along both $a$ and the $b$ axis (space group $I b c a)$. Frequently in the low crystallization temperature packing defects is introduced due to the irregular left-right packing leading to lattice disorder, which is called a limitdisordered structure (space group Pcaa). One can see from Fig. 5 that all the reflections show that the sPP formed a limit-disordered structure of the most stable form I. With the application of shear and the increase of shear rate, the peak positions remain unchanged indicating the crystalline form of the sPP sample has not been changed.

2D WAXD has been usually used to estimate the orien-

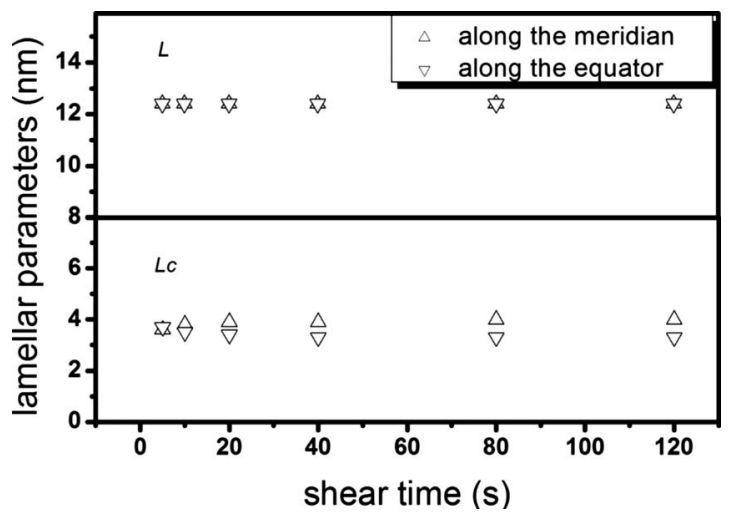

FIG. 10. Lamellae parameters: Long period $(L)$, crystal lamellar thickness $(L c)$ obtained from SAXS data of SPP after isothermal crystallization at $100{ }^{\circ} \mathrm{C}$ with different shear duration under fixed shear rate $5 \mathrm{~s}^{-1}$. 


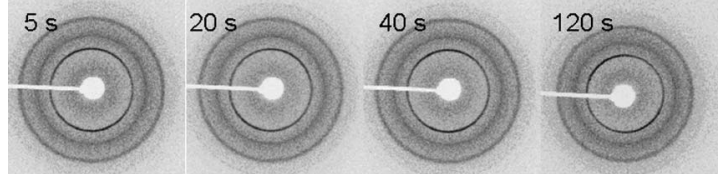

(a)

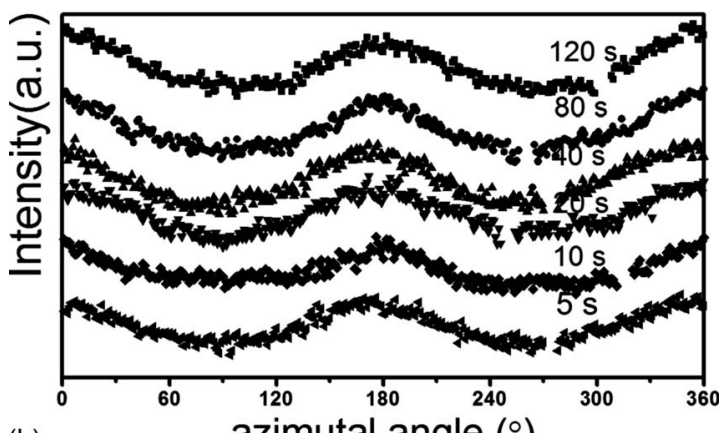

(b) azimutal angle $\left(^{\circ}\right)$

FIG. 11. (a) Selected 2D WAXD patterns and (b) azimuthal intensity curves of (200) reflections for sPP after isothermal crystallization at $100{ }^{\circ} \mathrm{C}$ applying a certain shear intensity $5 \mathrm{~s}^{-1}$ with different shear durations.

tation of crystallites. There is difference between the crystalline orientation detected by WAXD and lamellar orientation determined by SAXS because of the different structural scales observed by the two techniques. Some 2D WAXD patterns of sPP with variable shear rate and detailed intensity distribution of the (200) reflection were analyzed to study shear-induced crystallite orientation, as shown in Fig. 6. There show three full Debye rings of (200), (020), and (121) reflections of the crystal lattice planes from inside to outside of the images in Fig. 6(a). As shown in the image obtained for sample without shearing, there is no orientation of the crystallite, i.e., the intensity of reflection for every Debye ring is uniform. With the increase of the shear rate, WAXD patterns show arcs meaning the increasing anisotropy which is especially obvious in (200) reflection. When the shear rate reached $20 \mathrm{~s}^{-1}$, the (200) reflection crystalline ring is clearly concentrated which indicates the crystallites are highly oriented. From Fig. 6(b) the degree of variation in the intensity of reflections as a function of azimuthal angle is an indication of the orientational degree and one observes that orientation becomes more distinct with the increasing shear rate.

Much research has been done on the relaxation in sheared polymer melt including experiment and theory. The results showed that the polymer relaxation is much faster than polymer crystallization after shearing, especially at high temperature. One can image the shear effects on the melting polymers. If polymer melt is random coil, the random coil would be stretched by the applied shearing. The mark of shearing left on the final structure of sPP would be found from molecular chains to lamellae. If polymer melt is preordered structure, the effects of shearing on the final structure of sPP would be beyond the scale of the preordered structure. In our experiment, shear field has strong influence on the orientation of lamellae but the lamellar parameters $(L, L c)$ are independent on shear effects. One can deduce that before shear composed there should be mesomorphic structure, shear makes the orientation of mesophase which finally develops into oriented lamellae. Shear makes for the strong oriented lamellae, whereas the effect due to shear on the orientation of crystallite structure is relatively weaker which can be assessed by Herman's orientation function. ${ }^{74}$ Strobl $e t$ al. considered that the mesophase layer is composed of stretched sequences and the stretching is not perfect. A continuous rearrangement of the chain sequences will happen inside the mesophase during the thickening process. Our shearing results indicate that shear made a more influence on the lamellar orientation than crystallite orientation which supports Strobl's theory. Strobl's scenario may comprise an idea that the transient intermediate phase might exert a certain influence on the structure of the final product, e.g., lamellar thickness and orientation structure may reflect a memory of that mesomorphic phase. Figure 7 shows the schematic pictures with reference to Ref. 25 of crystallization process from a mesomorphic layer to a lamellar layer after shear field composed on the metastable mesophase stage. One can see that the intermediate phase would be the initial structure for polymer crystallite. Before shear composed it shows the isotropic mesomorphic structure where stable mesophase composed of folded chains does not have orientation. With shear intensity growing, the orientation of blocks would happen which can induce the high orientation of final lamellar.

To further study the stability of the mesophase, the crystallization of sPP under fixed shear rate $\left(5 \mathrm{~s}^{-1}\right)$ with different shearing times $(5,10,20,40,80$, and $120 \mathrm{~s})$ was performed. The 2D SAXS patterns for sPP isothermal crystallized at $100{ }^{\circ} \mathrm{C}$ are shown in Fig. 8. As the shear duration increased, the SAXS intensity became stronger along the meridian and weaker along the equator, as shown in Fig. 9. Detailed parameters about lamellar structure in both directions are shown in Fig. 10. The long period was not influenced by shear duration under this crystallization condition. The lamellar thickness along the meridian increased with the increasing of the shear duration, whereas it decreases along the equator with the shear duration decreasing. There is no variation of the orientation degree with the enhancement of shear duration, meaning that shear duration have no influence on orientation of mesomorphic blocks, i.e., the mesophase is rather stable at $100{ }^{\circ} \mathrm{C}$. Under certain shear intensity, the

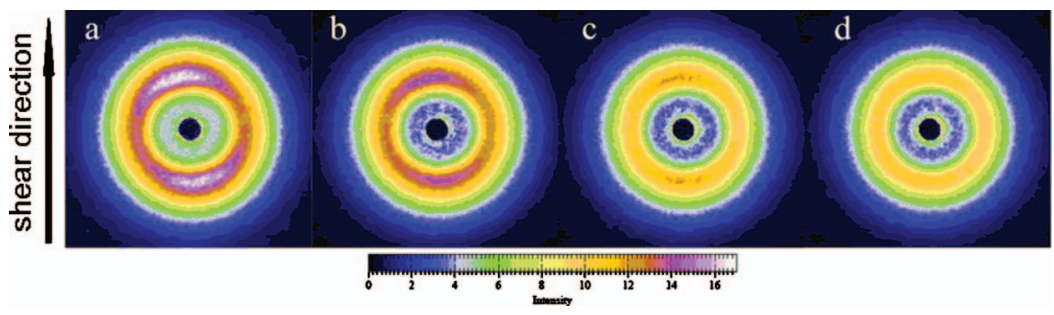

FIG. 12. (Color) 2D SAXS patterns of sPP sheared at different temperature: (a) $100{ }^{\circ} \mathrm{C}$, (b) $110^{\circ} \mathrm{C}$, (c) $120^{\circ} \mathrm{C}$, and (d) $180{ }^{\circ} \mathrm{C}$ and then isothermal crystallization at $100{ }^{\circ} \mathrm{C}$. 


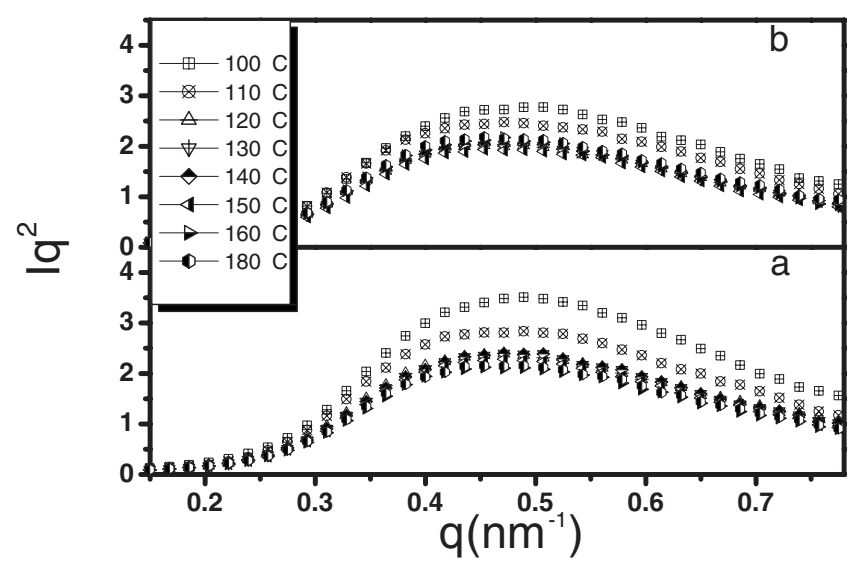

FIG. 13. $I q^{2}$ vs scattering vector $q$ of sPP isothermal crystallization at $100{ }^{\circ} \mathrm{C}$ after sheared at different temperatures. (a) Along the meridian; (b) along the equator.

extension of folding chain would be confined within the mesophase and the balance between chain extension and relaxation is fixed which is not disturbed by the shear duration. The metastable mesophase composed of folded chains will have orientation along the shear direction which would proceed to grow both laterally during the initial crystallization. For the ordered phase to crystallize from the amorphous melt, some chain segments around folded chains must be somehow influenced by the shear duration which would increase the thickness of mesomorphic layer. The decrease in lamellar thickness along the equator must be attributed to the transfer of chain segments forming the mesomorphic structure from the direction along the equator to the direction along the meridian. Figure 11 shows the 2D WAXD patterns showing orientation structure of sPP under this crystallization condition. All the patterns show anisotropy in (200) reflection and with shear duration increasing there is no obvious distinction, as shown by azimuthal intensity curves of (200) reflections. It approved the stability of the mesophase during SPP crystallization process together with the SAXS data.

In order to investigate the temperature dependence of the stability of the mesophase, the samples were sheared with shear rate of $5 \mathrm{~s}^{-1}$ at different temperatures (100, 110, 120, $130,140,150,160$, and $\left.180{ }^{\circ} \mathrm{C}\right)$, then cooled to crystallization temperature of $100{ }^{\circ} \mathrm{C}$ and kept for a certain time. The 2D SAXS patterns and Lorentz corrected intensity $I q^{2}$ versus scattering vector $q$ are shown in Figs. 12 and 13. As the shear temperature increases, the patterns show a decreasing degree of anisotropy of the crystalline distribution and the intensity from SAXS becomes lower with the increase in the shear temperature. Figure 14 shows the detailed structure parameters in both directions. The long period still does not have any change. The lamellar thicknesses along the meridian and along the equator all have little changes with increasing shear temperature. From the results we can see that stabilization of orientational mesophase rely on shear temperature. With shear temperature increasing, the orientation of mesophase relaxed which directly made for the isotropy of final crystallinity. Figure 15 shows 2D WAXD patterns and azimuthal intensity curves of (200) reflections of sPP under dif-

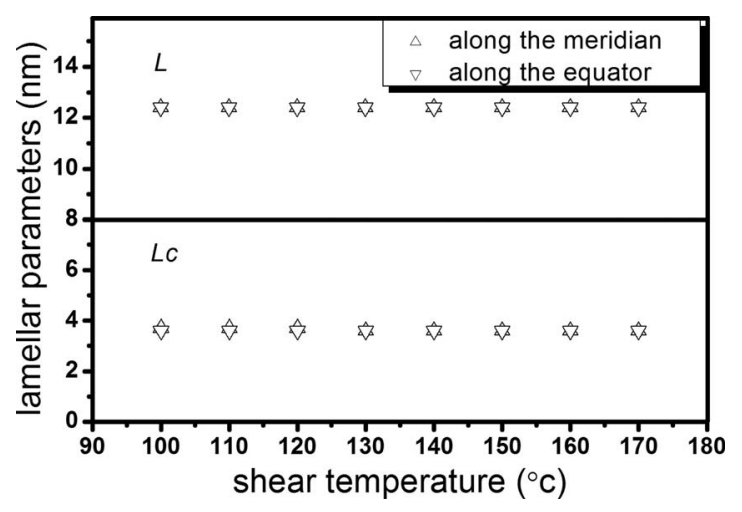

FIG. 14. Lamellae parameters: Long period $(L)$, crystal lamellar thickness $(L c)$ obtained from SAXS data of sPP with different shear temperatures and then isothermal crystallization at $100{ }^{\circ} \mathrm{C}$.

ferent shear temperature. The patterns show decreasing anisotropy in (200) reflection with shear temperature increasing. The variation of the orientational behavior of (200) reflection as indicated by WAXD is consistent with SAXS results, which all approved the reliance of the orientational mesophase on shear temperature.

\section{CONCLUSIONS}

In polymer processing, shear field induces the orientation of chains in melt and influence the subsequent crystallization development. A short shear pulse is deemed to be a good method to study the initial crystallization process. The shear is imposed on melt for a fixing shear time which is relatively shorter than the entire crystallization time. During shear, the crystallization growth can be even ignored and the effect of shear on the initial crystallization can be isolated studied. In this paper, the crystalline form of the sPP does not transform under different shear conditions but the orienta-

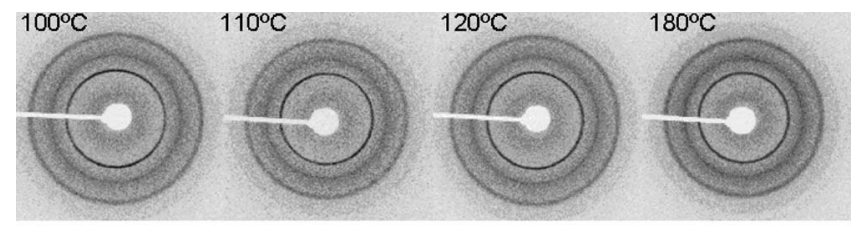

(a)

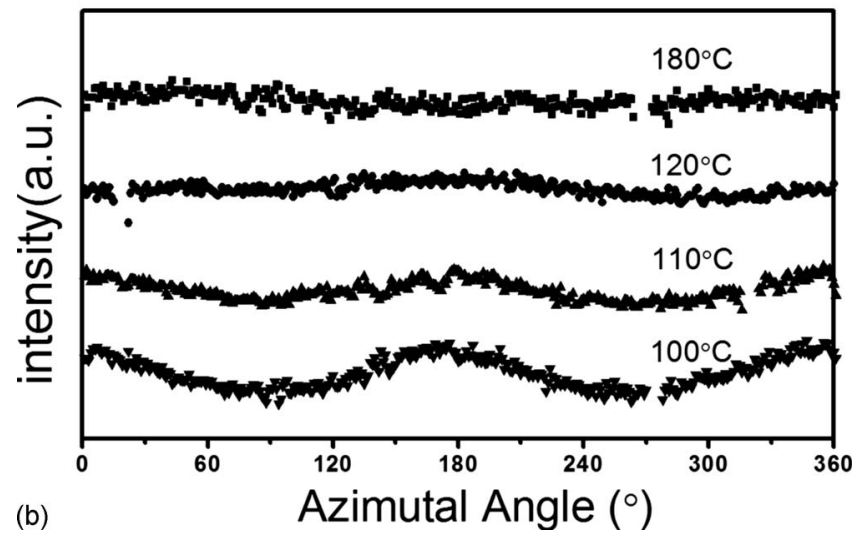

FIG. 15. (a) Selected 2D WAXD patterns and (b) azimuthal intensity curves of (200) reflections of sPP isothermal crystallization at $100{ }^{\circ} \mathrm{C}$ after sheared at different temperatures. 
tional degree of lamellae and the crystalline lattice plane (200) are dependent on the shear rate, especially the lamellar orientation is more obvious. Lamellar thickness and long period have little changes with increasing shear rates. Along with longer shear time, the long period also remains at a fixed value but the lamellar thickness along the meridian and the equator have a little variation especially with shorter shear time, and then fix with further increasing shear time, which means that a balance has been obtained. We found that higher shear temperature offers chances for chain relaxation. Shear has no influence on superstructure of sPP under higher shear temperature $\left(>130{ }^{\circ} \mathrm{C}\right)$. It may be indicated that there exists a mesomorphic structure before the shearing and the initial crystallization which further develop into lamellae. The long period and the crystallite size have not been affected by the shear but the preorder structure arraying regularly induced the occurrence of orientation. We think that the final structure of SPP in the shear is determined by the evolution of the sheared mesophase.

\section{ACKNOWLEDGMENTS}

This work was supported by the National Natural Science Foundation of China (Grant Nos. 20574069, 50773082, and 20490220) Programs, the Fund for Creative Research Groups (Grant No. 50621302), and subsidized by the Special Funds for National Basic Research Program of China (Grant No. 2003CB615600). SAXS experiments were supported by HASYLAB project (No. II20052011) and KEK project (No. 2006G131).

${ }^{1}$ J. D. Hoffman and J. I. Lauritzen, Jr., J. Res. Natl. Bur. Stand. 65A, 297 (1961).

${ }^{2}$ K. Armitstead and G. Goldbeck-Wood, Adv. Polym. Sci. 100, 219 (1992).

${ }^{3}$ J. J. Point, M. Dosière, Polymer 30, 2292 (1989).

${ }^{4}$ D. M. Sadler, Nature (London) 326, 174 (1987).

${ }^{5}$ G. Kanig, Colloid Polym. Sci. 261, 373 (1983).

${ }^{6}$ G. Kanig, Colloid Polym. Sci. 269, 1118 (1991).

${ }^{7}$ K. Tashiro, S. Sasaki, N. Gose, and M. Kobayashi, Polymer 30, 485 (1998).

${ }^{8}$ T. Okada, H. Saito, and T. Inoue, Macromolecules 25, 1908 (1992).

${ }^{9}$ P. D. Olmsted, W. C. K. Poon, T. C. B. McLeish, T. C. B. Terrill, and A. Ryan, Phys. Rev. Lett. 81, 373 (1998).

${ }^{10}$ N. V. Pogodina, S. K. Siddiquee, J. W. Van Egmond, and H. H. Winter, Macromolecules 32, 1167 (1999).

${ }^{11}$ B. S. Hsiao, Z. G. Wang, F. Yeh, Y. Gao, and K. C. Sheth, Polymer 40, 3515 (1999).

${ }^{12}$ Z. G. Wang and B. S. Hsiao, Macromolecules 33, 978 (2000).

${ }^{13}$ K. Fukao and Y. Miyamoto, Phys. Rev. Lett. 79, 4613 (1997).

${ }^{14}$ G. Matsuba, K. Kaji, K. Nishida, T. Kanaya, and M. Imai, Macromolecules 32, 8932 (1999).

${ }^{15}$ M. Imai, K. Mori, T. Mizukami, K. Kaji, and T. Kanaya, Polymer 33 4457 (1992).

${ }^{16}$ G. Matsuba, T. Kanaya, M. Saito, K. Kaji, and K. Nishida, Phys. Rev. E 62, R1497 (2000).

${ }^{17}$ G. Matsuba, K. Kaji, K. Nishida, T. Kanaya, and M. Imai, Polymer 31, 722 (1999).

${ }^{18}$ M. Imai, K. Kaji, T. Kanaya, and Y. Sakai, Phys. Rev. B 52, 12696 (1995).

${ }^{19}$ W. Z. Ostwald, Phys. Chem. (Munich) 22, 286 (1897).

${ }^{20}$ G. Strobl, The Physics of Polymers, 1st ed. (Springer, Berlin, 1997), Chap. 4.

${ }^{21}$ G. Hauser, J. Schmidtke, and G. Strobl, Macromolecules 31, 6250 (1998).
${ }^{22}$ B. Heck, T. Hugel, M. Iijima, E. Sadiku, and G. Strobl, New J. Phys. 1, 17.1 (1999).

${ }^{23}$ M. Iijima and G. Strobl, Macromolecules 33, 5204 (2000).

${ }^{24}$ R. Graf, A. Heuer, and H. W. Spiess, Phys. Rev. Lett. 80, 5738 (1998).

${ }^{25}$ G. Strobl, Eur. Phys. J. E 3, 165 (2000).

${ }^{26}$ Q. Fu, B. Heck, and G. Strobl, Macromolecules 34, 2502 (2001).

${ }^{27}$ V. B. F. Mathot, R. L. Scherrenberg, and T. F. J. Pijpers, Polymer 39, 4541 (1998).

${ }^{28}$ G. H. Michler, in Kunststoff-Mikromechanik, edited by C. Hanser (Springer-Verlag, Berlin, 1992), p. 187.

${ }^{29}$ D. C. Bassett and D. Patel, Polymer 35, 1855 (1994).

${ }^{30}$ N. Okui and T. Kawai, Makromol. Chem. 154, 161 (1972).

${ }^{31}$ J. Minick, A. Moet, A. Hiltner, E. Baer, and S. P. J. Chum, J. Appl. Polym. Sci. 58, 1371 (1995).

${ }^{32}$ T. Hugel, G. Strobl, and R. Thomann, Acta Polym. 50, 214 (1999).

${ }^{33}$ M. Grasruck and G. Strobl, Macromolecules 36, 86 (2003).

${ }^{34}$ B. Heck, T. Hugel, M. Iijima, and G. Strobl, Polymer 41, 8839 (2000).

${ }^{35}$ E. B. Sirota, Macromolecules 40, 1043 (2007).

${ }^{36}$ J. P. Fernández-Blázquez, J. Pérez-Manzano, A. Bello, and E. Pérez, Macromolecules 40, 1775 (2007).

${ }^{37}$ H. Y. Wen, S. C. Jiang, Y. F. Men, and L. J. An, Macromol. Chem. Phys. 209, 1721 (2008).

${ }^{38}$ J. A. Ewen, R. L. Jones, A. Razavi, and J. D. Ferrara, J. Am. Chem. Soc. 110, 6255 (1988)

${ }^{39}$ J. A. Ewen, M. J. Elder, R. L. Jones, L. Haspeslagh, J. L. Atwood, S. G. Bott, and K. Robinson, Makromol. Chem., Macromol. Symp. 48/49, 253 (1991).

${ }^{40}$ A. J. Lovinger and D. D. Davis, Macromolecules 24, 552 (1991).

${ }^{41}$ A. J. Lovinger and B. Lotz, J. Polym. Sci., Part B: Polym. Phys. 35, 2523 (1997).

${ }^{42}$ R. A. Phillips and R. L. Jones, Macromol. Chem. Phys. 200, 1912 (1999).

${ }^{43}$ Z. G. Wang, X. H. Wang, and B. S. Hsiao, J. Polym. Sci., Part B: Polym. Phys. 39, 2982 (2001).

${ }^{44}$ P. Supaphol, J. Appl. Polym. Sci. 82, 1083 (2001).

${ }^{45}$ T. Kawai and G. Strobl, Macromolecules 37, 2249 (2004).

${ }^{46}$ J. Schmidtke, G. Strobl, and T. Thurn-Albrecht, Macromolecules 30, 5804 (1997).

${ }^{47}$ W. Zhou, S. Z. D. Cheng, S. Putthanarat, R. K. Eby, D. H. Reneker, B. Lotz, S. Magonov, E. T. Hsieh, R. G. Geerts, S. J. Palackal, G. R. Hawley, and R. B. Welch, Macromolecules 33, 6861 (2000).

${ }^{48}$ A. Eckstein, J. Suhm, C. Friedrich, R. D. Maier, J. Sassmannshausen, M. Bochmann, and R. Mulhaupt, Macromolecules 31, 1335 (1998).

${ }^{49}$ K. Kobayashi and J. Nagasawa, J Macromol Sci., Phys. 4, 331 (1970).

${ }^{50}$ A. Wereta and C. Gogos, Polym. Eng. Sci. 11, 19 (1971).

${ }^{51}$ R. H. Somani, L. Yang, and B. S. Hsiao, Macromolecules 35, 9096 (2002).

${ }^{52}$ H. Huo, S. C. Jiang, and L. J. An, Macromolecules 37, 2478 (2004).

${ }^{53}$ F. Auriemma and C. De Rosa, Macromolecules 39, 7635 (2006).

${ }^{54}$ L. B. Li and W. H. De Jeu, Phys. Rev. Lett. 92, 075506 (2004).

${ }^{55}$ H. N. An, B. J. Zhao, Z. Ma, C. G. Shao, X. Wang, Y. P. Fang, L. B. Li, and Z. M. Li, Macromolecules 40, 4740 (2007).

${ }^{56}$ A. Hammersley, Computer Program FIT2D, Version V12.012, ESRF, 1998.

${ }^{57}$ G. R. Strobl and M. Schneider, J. Polym. Sci., Part B: Polym. Phys. 18, 1343 (1980).

${ }^{58}$ R. K. Verma and B. S. Hsiao, Trends Polym. Sci. 4, 312 (1996).

${ }^{59}$ Z. G. Wang, B. S. Hsiao, B. B. Sauer, and W. G. Kampert, Polymer 40, 4615 (1999).

${ }^{60}$ R. K. Verma, H. Marand, and B. Hsiao, Macromolecules 29, 7767 (1996).

${ }^{61}$ D. Y. Tsvankin, Y. A. Zubov, and A. I. Kitaigorodskii, J. Polym. Sci., Part C. 16, 4081 (1968).

${ }^{62}$ W. Ruland, J. Appl. Crystallogr. 4, 70 (1971).

${ }^{63}$ N. S. Murthy, C. Bednarczyk, R. A. F. Moore, and D. T. Grubb, J. Polym. Sci., Part B: Polym. Phys. 34, 821 (1996).

${ }^{64}$ G. Strobl, The Physics of Polymers, 2nd ed. (Springer, Berlin, 1997).

${ }^{65}$ C. De Rosa and P. Corradini, Macromolecules 26, 5711 (1993).

${ }^{66}$ P. Sozzani, R. Simonutti, and M. Galimberti, Macromolecules 26, 5782 (1993).

${ }^{67}$ B. Lotz, A. J. Lovinger, and R. E. Cais, Macromolecules 21, 2375 (1988).

${ }^{68}$ A. J. Lovinger, B. Lotz, D. D. Davis, and F. J. Padden, Macromolecules 
26, 3494 (1993).

${ }^{69}$ C. De Rosa, F. Auriemma, and V. Vinti, Macromolecules 31, 7430 (1998).

${ }^{70}$ G. Natta, M. Peraldo, and G. Allergra, Makromol. Chem. 75, 215 (1964).

${ }^{71}$ Y. Chatani, H. Maruyama, T. Asanuma, and T. Shimmura, J. Polym. Sci., Part B: Polym. Phys. 28, 393 (1990).
${ }^{72}$ Y. Chatani, H. Maruyama, T. Asanuma, and T. Shimmura, J. Polym. Sci., Part B: Polym. Phys. 29, 1649 (1991).

${ }^{73}$ P. Sozzani, R. Simonutti, and A. Comotti, Macromol. Symp. 89, 513 (1995).

${ }^{74}$ J. J. Hermans, P. H. Hermans, D. Vermeas, and A. Weidinger, Rec. Trav. Chim. 65, 427 (1946). 